\title{
Designing of Dynamic Sensor Networks based on Meter-range Swarming Flight Type Air Nodes
}

\author{
Chul-Gyu Kang, Dae-Hwan Kim, Member, KIICE
}

\begin{abstract}
Dynamic sensor network(DSN) technology which is based on swarming flight type air node offers analyzed and acquired information on target data gathered by air nodes in rotation flight or 3 dimension array flight. Efficient operation of dynamic sensor network based on air node is possible when problems of processing time, data transmission reliability, power consumption and intermittent connectivity are solved. Delay tolerant network (DTN) can be a desirable alternative to solve those problems. DTN using storeand-forward message switching technology is a solution to intermittent network connectivity, long and variable delay time, asymmetric data rates, and high error rates. However, all processes are performed at the bundle layer, so high power consumption, long processing time, and repeated reliability technique occur. DSN based on swarming flight type air node need to adopt store-andforward message switching technique of DTN, the cancelation scheme of repeated reliability technique, fast processing time with simplified layer composition.
\end{abstract}

Index Terms - Dynamic Sensor Network, Air Node, Ubiquitous Sensor Network, Delay Tolerant Network

\section{INTRODUCTION}

RECENTLY, a lot of researchers started to have strong interest in and are actively carrying out studies on USN(Ubiquitous Sensor Network) technology, which makes it possible to manage and acquire information by being connected to a network in real time and read information on the surrounding environments as well as on all surrounding objects from sensors installed in all places [1]. Nevertheless, most of the studies are focused on a static sensor network and low data rates services such as home automation, appliances control, lamp control, home security, and so on. Static and low data rate networks are not able to quickly respond natural disasters like the Fukushima Daiichi nuclear disaster, which had been caused from the Tohoku earthquake and tsunami on 11 March 2011, and the Chernobyl disaster, which had

\footnotetext{
Manuscript received November 17, 2011; revised November 28, 2011; accepted December 5, 2011.

Chul-Gyu Kang is with the Department of Communication, Korea Electronics Technology Institute, Seoungnam-si, Gyeonggi-do, 463-816, Korea (Email: chgkang@keti.kr)

Dae-Hwan Kim is with the Department of Communication, Korea Electronics Technology Institute, Seoungnam-si, Gyeonggi-do, 463-816, Korea (Email:kimdh@keti.kr)
}

happened on 26 April 1986[2],[3]. In addition, even if sensors gather information on objects in the surroundings, it is very difficult that such gathered information is rapidly transferred to a remote server or a manager located away from the site in many types. In order to solve those problems, it is absolutely required to develop a dynamic sensor network based on swarming-flight-type air nodes.

Dynamic sensor network(DSN) technology is based on air nodes that offer analyzed information obtained from targets in rotation flight or 3 dimension array flight. Air nodes fly, sense, and return on a specific time period basis, so that efficient management of battery or sensor exchange of a sensor node is possible and risks such as loss of or damage to air nodes are reduced. Moreover, DSN technology makes it possible to prevent human losses, act rapidly on searches or rescues and carry out military actions in places where static sensor nodes and monitoring equipment cannot carry out their own duties at important facilities, urban areas, disaster areas, areas exposed to radioactivity, fire areas, and military operation areas.

In order to run dynamic sensor networks based on air nodes in an efficient way, matters like slow processing speed, low reliability, high power consumption, and intermittent connectivity must be solved first. Air nodes change their won formation and track according to given conditions actively and perform their designated missions. As a result, intermittent connectivities of networks are periodically happen between each air node. Even if connection and disconnection of networks occur repeatedly, all the transmitted data must be keep in fact. Possibly, communication technology which makes devices operate in low power mode is required because excessive power consumption causes air nodes crash and that leads to a failed mission. Air nodes should perform aviation and communication missions at the same time using limited amounts of power. Fast processing time and transmission reliability have to be ensured as air nodes create their network and appropriate formation in consideration of conditions in transmitting and receiving information each other.

For this reason, "delay tolerant network" technology is thought to be a good and desirable alternative. Delay tolerant networks equipped with store-and-forward message switching technology can transmit data without any loss of them in places where network connection and disconnection occur repeatedly. Therefore, DTN is 
necessary in environments in which network topology changes are made dynamically.

\section{DELAY TOLERANT NETWORK}

\section{A. Packet Switching Network}

Communication via the Internet is based on packet switching. Each packet that makes up a message can take a different path through the network. If one link is disconnected, a packet takes another link. The usability of the Internet depends on several important assumptions[4],[5].

- Continuous, bidirectional end-to-end path: A continuously available bidirectional connection between source and destination to support end-to-end interaction

- Short round-trips: Small and relatively consistent network delay in sending data packets and receiving the corresponding acknowledgement packets.

- Symmetric data rates: Relatively consistent data rates in both directions between source and destination.

- Low error rates: Relatively little loss or corruption of data on each link.

\section{B. Delay Tolerant Network}

A delay tolerant network is part of a regional network. It is an end-to-end architecture providing communications in and through highly stressed environments. Stressed networking environments include those with intermittent connectivity, large or variable delays, asymmetric data rate, and high bit error rates[6]-[8].

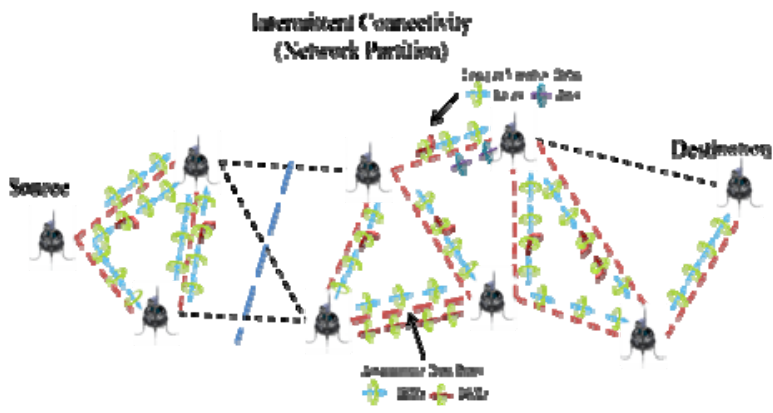

Fig. 1. Stressed networking environments.

- Intermittent connectivity : If there is no end-to-end path between source and destination, called network partitioning, end-to-end communication using the TCP/IP protocols does not work. Other protocols are required.

- Long and variable delay : In addition to intermittent connectivity, long propagation delays between nodes and variable queuing delays at nodes contribute to end-to-end path delays that can defeat Internet protocols and applications that relay on quick return of acknowledgements or data.

- Asymmetric data rates: The Internet supports moderate asymmetries of bidirectional data rate for users with cable TV or asymmetric DSL access. But if asymmetries are large, they defeat conversational protocol.

- High error rates: Bit errors on links require correction or retransmission of the entire packet. For a given link-error rate, fewer retransmissions are needed for hop-by-hop than for end-to-end retransmission.

Delay tolerant networks overcome the problems associated with intermittent connectivity, long and variable delay, asymmetric data rates, and high error rates by using store-and-forward message switching. Whole messages, entire blocks of application program user data, or pieces of such messages are moved from a storage place on one nodes to a storage place on another node, along a path that eventually reaches the destination. A message, once transmitted, may need to be retransmitted if an error occurs at an upstream(toward the destination) air node or link, or if an upstream air node declines acceptance of a forwarded message.

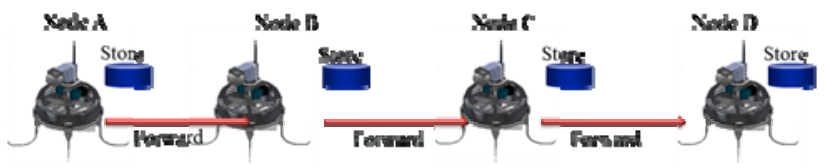

Fig. 2. Store-and-forward message switching.

Delay tolerant network architecture implements storeand-forward message switching by overlaying a new protocol layer, called the bundle layer, above the transport layers of the networks on which it is hosted and below applications. The bundle layer forms an overlay that employs persistent storage to help combat network interruption.
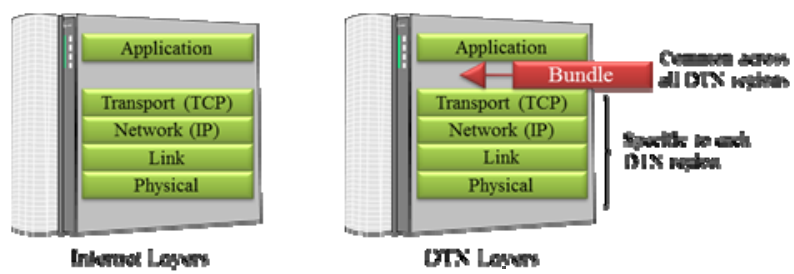

Fig. 3. Bundle layer architecture for the DTN.

The bundle layer supports node-to-node retransmission by means of custody transfers for delay tolerant network. Such transfers are arranged between the bundle layers of successive nodes at the initial request of the source application. A bundle custodian, which is a node receiving application data units, must store a data until 
either another node accepts custody, or expiration of the bundle's time-to-live, which is intended to be much longer than a custodian's time-to-acknowledge. Therefore, custody transfers do not provide guaranteed end-to-end reliability.

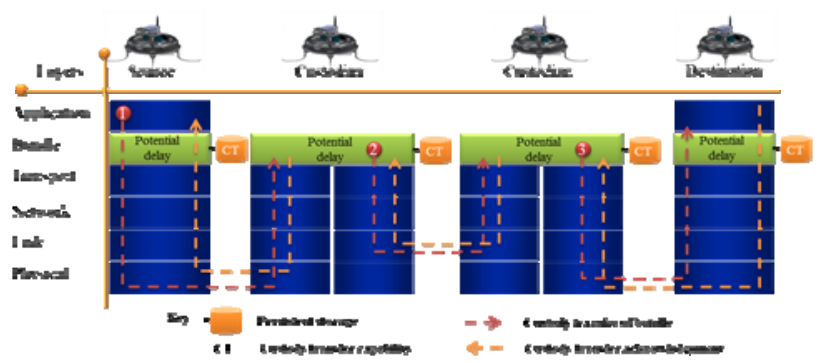

Fig. 4. Reliability guarantee technique for data transmission of DTN.

\section{Problem of Delay Tolerant Network}

Delay tolerant network resolves the problems related with intermittent connectivity, long and variable delay, asymmetric data rates, and high error rates by using storeand-forward message switching with a persistent storage at the bundle layer. However, it has the problems associated with repeated reliability techniques, long delay time, high processing time, and high power consumption[9].

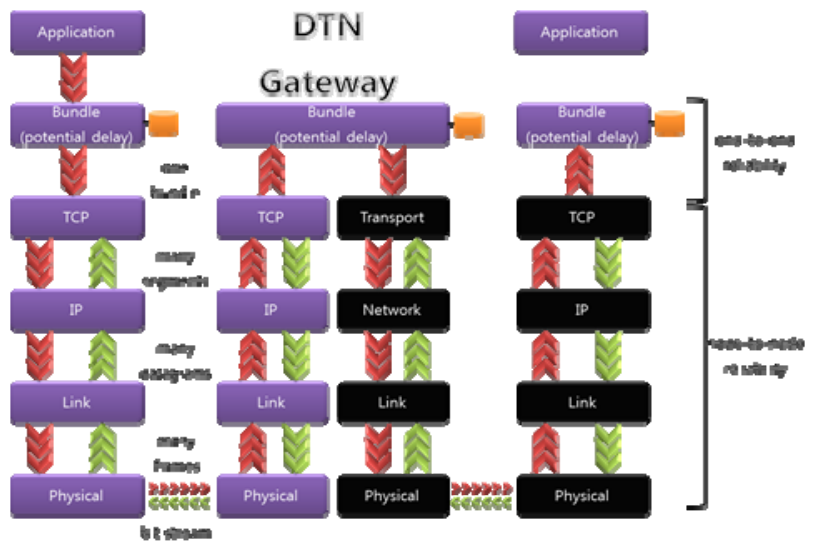

Fig. 5. Data processing procedure of DTN.

- Repeated reliability techniques : The bundle layer supports hop-to-hop retransmission by means of custody transfers. When the current bundle layer custodian sends a bundle to the next node, it requests a custody transfer and starts a time-to-acknowledge retransmission timer. If the next-hop bundle layer accepts custody, it returns a acknowledgement to the sender. If no acknowledgement is returned before the sender's time-to-acknowledge expires, the sender retransmits the bundle. However, HDLC(high-level data link control) protocol and PPP(point-to-point protocol) of the data-link layer have the similar mechanism with custody transfers. For example, when a frame of HDLC or PPP is transmitted, a timer of the transmitted signal is started. If acknowledgement is not received before its timer expires, retransmission is executed.

- Long delay time : All of the received data are stored and processed at the bundle layer. The bundle layer locates above the transport layer, so that all of the lower layers must deliver the received data to the bundle layer, and then wait until the received data is processed and stored by the bundle layer. This procedure makes the processing time longer.

- Longer processing time and large power consumption : The bundle layer stores all of received data in its own storage place and process them. To process and store such received data, all of lower layers must decasualize the received data by their own procedures then deliver them to the upper layer because the bundle layer locates above the transport layer. The same procedure applies to all air nodes in the way to the destination air node. Consequently, the longer data process time and large power consumption are caused by that procedure.

\section{SUGGESTIONS}

The delay tolerant network processes all received data at the bundle layer. Therefore, that requires a long processing time, large power consumption, long delay time, and also reliability problems can occur repeatedly. In this document, we suggest that a dynamic sensor network based on air nodes adopts store-and-forward message switching technique of the delay tolerant network, and the storage place is located in DSN IP layer.



Fig. 6. Dynamic sensor network(DSN) layers.

- Store-and-forward message switching : It is necessary to apply a permanent storage device to DSN for handling intermittent network connectivities which occur due to the movement of air nodes. If this storage device is located on the upper layer, processing time and storing time is delayed, so a flash memory used at the network layer is exchanged to a persistent storage device. From this change, store-and-forward message switching is able to support dynamic sensor network.

- Reliability techniques : The reliability techniques of the delay tolerant network that are similar to those of the 
data-link layer and the network layer are removed, and the reliability techniques that each layer support are made full use of the techniques. In addition, a function of diagnosing the current network status is adopted to ensure successful data transmission.

- Simplified layer composition : In case of considering with respect to power consumption and processing time of air nodes, the network layer composition must be simplified. Since the storage place is moved into the network layer, inserting the bundle layer to the DSN layer is unnecessary. The function of storing data is operated at the network layer so that unnecessary data transmission and processing can be prevented. The problems of power consumption and data processing time of dynamic sensor network based on air node can be solved as well.

\section{CONCLUSION}

Dynamic sensor network(DSN) technology which is based on swarming flight type air node offers analyzed and acquired information on target data gathered by air nodes in rotation flight or 3 dimension array flight. Efficient operation of dynamic sensor network based on air node is possible when problems of processing time, data transmission reliability, power consumption and intermittent connectivity are solved.

Delay tolerant network can be a desirable alternative to solve those problems. Delay tolerant network using storeand-forward message switching technology is a solution to intermittent network connectivity, long and variable delay time, asymmetric data rates, and high error rates. However, all processes are performed at the bundle layer, so high power consumption, long processing time, and repeated reliability technique occur.

Dynamic sensor network based on swarming flight type air node needs to adopt store-and-forward message switching technique of delay tolerant network, the cancelation scheme of repeated reliability technique, fast processing time with simplified layer composition. Therefore, DSN can offer many types of services like natural disaster or environment pollution monitoring, and as a result, it will bring enormous profits to service providers.

\section{ACKNOWLEDGMENT}

This work was financially supported by the grant from the Industrial Source Technology Development Program 10039988 of the Ministry of Knowledge Economy of Korea and Korea Evaluation Institute of Industrial Technology.

\section{REFERENCES}

[1] Hyunjae Lee, Seungheui Jeong and Changheon Oh, "A study on the high precision location estimation scheme for RTLS services," SKTelecom Telecommunications Review, Vol. 18, No. 6, pp. 10721088, October 2008.

[2] Wikipedia.

http://ko.wikipedia.org/wiki/http://en.wikipedia.org/wiki/Fukushim a Daiichi_nuclear_disaster

[3] http://en.wikipedia.org/wiki/Chernobyl_disaster

[4] Will Ivancic, Phil Paulsen, Dave Stewart, John Taylor, Scott Lynch, Jay Heberle, James Northam, Chris Jackson and Lloyd Wood, "Large file transfers from space using multiple ground terminals and delay-tolerant networking," IEEE Global Communications Conference (Globecom 2010), Miami, Florida, December 2010.

[5] Giorgos Papastergiou, Ioannis Psaras and Vassilis Tsaoussidis, "Deep-space transport protocol: a novel transport scheme for space DTNs computer communications (COMCOM)," Elsevier Science, Special Issue on Delay-/Disruption-Tolerant Networking, Volume 32, Issue 16, pp. 1757-1767, October 2009.

[6] DTN Research Group. http://www.dtnrg.org/

[7] V. Cerf et. al., "Delay-Tolerant Network Architecture", RFC 4838, April 2007.

[8] K. Scott and S. Burleigh, "Bundle Protocol Specification", RFC 5050, Novemberl 2007.

[9] M. Ramadas et al., Licklider Transmission Protocol-Specification, IETF RFC 5326, experimental, September 2008.

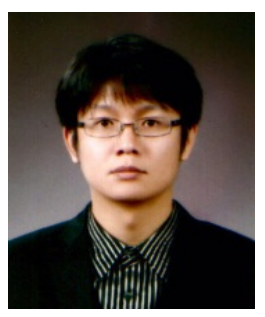

Chul Gyu Kang was born in Daejoen, Korea, on January 31,1976 . He received the B. S. and M.S.E. degrees in information technology engineering from Korea University of Technology and Education, Chungnam, Korea, in 2004 and 2006, respectively. He is a Ph.D. student in information technology engineering from Korea University of Technology and Education, Chungnam, Korea. His research interests are in the areas of wireless communications and wireless sensor networks with particular emphasis on wireless localization

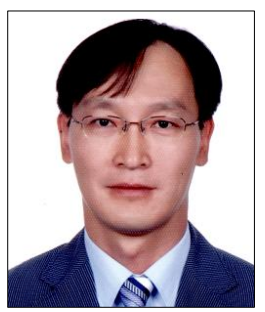

Dae-Hwan Kim received the B.S. and M.S.E degrees in electronics engineering from Myong Ji University, Gyeonggi, Korea, in 1991 and 1993, respectively. He is working as a principal research engineer at RFID/USN convergence research center convergence Emerging Industries $\mathrm{R} \& \mathrm{D}$ division, Korea Electronics Technology Institute. He is a Ph.D student in electronics engineering from Ajou University, Gyeonggi, Korea. His research interests are RFID/USN and embeded system. 